\title{
Protein intake and lumbar bone density: the Multi-Ethnic Study of Atherosclerosis (MESA)
}

\author{
Tian $\mathrm{Hu}^{1}$, Nahid J. Rianon ${ }^{2}$, Jennifer A. Nettleton ${ }^{3}$, Joseph A. Hyder ${ }^{4}$, Jiang He ${ }^{1}$, Lyn M. Steffen ${ }^{5}$, \\ David R. Jacobs $\mathrm{Jr}^{5}$, Michael H. Criqui ${ }^{6}$ and Lydia A. Bazzano ${ }^{1 *}$ \\ ${ }^{1}$ Department of Epidemiology, Tulane University School of Public Health and Tropical Medicine, 1440 Canal Street, \\ SL-18, Suite 2000, New Orleans, LA 70112, USA \\ ${ }^{2}$ Department of Family and Community Medicine, University of Texas Medical School, Houston, TX, USA \\ ${ }^{3}$ Division of Epidemiology, Human Genetics and Environmental Sciences, University of Texas Health Science Center \\ School of Public Health, Houston, TX, USA \\ ${ }^{4}$ Department of Anesthesiology, Mayo Clinic, Rochester, MN, USA \\ ${ }^{5}$ Division of Epidemiology and Community Health, University of Minnesota School of Public Health, Minneapolis, MN, USA \\ ${ }^{6}$ Division of Preventive Medicine, Department of Family and Preventive Medicine, University of California, \\ San Diego, CA, USA
}

(Submitted 17 April 2014 - Final revision received 1 July 2014 - Accepted 9 July 2014 - First published online 5 September 2014)

\begin{abstract}
Dietary protein has been shown to increase urinary Ca excretion in randomised controlled trials, and diets high in protein may have detrimental effects on bone health; however, studies examining the relationship between dietary protein and bone health have conflicting results. In the present study, we examined the relationship between dietary protein (total, animal and vegetable protein) and lumbar spine trabecular volumetric bone mineral density (vBMD) among participants enrolled in the Multi-Ethnic Study of Atherosclerosis ( $n$ 1658). Protein intake was assessed using a FFQ obtained at baseline examination (2000-2). Lumbar spine vBMD was measured using quantitative computed tomography (2002-5), on average 3 years later. Multivariable linear and robust regression techniques were used to examine the associations between dietary protein and vBMD. Sex and race/ethnicity jointly modified the association of dietary protein with vBMD ( $P$ for interaction=0.03). Among white women, higher vegetable protein intake was associated with higher vBMD $(P$ for trend=0.03), after adjustment for age, BMI, physical activity, alcohol consumption, current smoking, educational level, hormone therapy use, menopause and additional dietary factors. There were no consistently significant associations for total and animal protein intakes among white women or other sex and racial/ethnic groups. In conclusion, data from the present large, multi-ethnic, populationbased study suggest that a higher level of protein intake, when substituted for fat, is not associated with poor bone health. Differences in the relationship between protein source and race/ethnicity of study populations may in part explain the inconsistent findings reported previously.
\end{abstract}

Key words: Lumbar bone mineral density: Dietary protein: Nutrition: Cohort studies

The principal complication of bone loss is fragility fracture. Approximately 44 million US adults aged 50 years and older ( $80 \%$ women) have low bone mass that puts them at risk for fragility fractures ${ }^{(1)}$. In 2005, more than 2 million new fragility fractures occurred in the USA, with direct health care costs totalling more than $\$ 17$ billion $^{(2)}$. Roughly $50 \%$ of those with hip fractures will never walk again without assistance and $25 \%$ will require long-term care ${ }^{(3)}$.

Protein-rich foods are often associated with higher levels of satiety than carbohydrate-rich foods ${ }^{(4)}$. Owing to this beneficial effect, diets that include protein-rich foods are often advocated for weight loss and weight maintenance ${ }^{(4)}$. However, dietary protein has unclear effects on bone health ${ }^{(5)}$. Short-term intervention studies using purified protein supplements have shown that $1 \mathrm{mg} \mathrm{Ca}$ is, on average, lost in the urine for every $1 \mathrm{~g}$ increase in protein intake $\mathrm{C}^{(6)}$. However, high-protein diets appear to increase intestinal $\mathrm{Ca}$ absorption, while low-protein diets decrease it ${ }^{(7)}$.

Observational epidemiological studies are inconsistent on the subject of dietary protein and the risk of fractures.

Abbreviations: BMD, bone mineral density; CT, computed tomography; MESA, Multi-Ethnic Study of Atherosclerosis; MET, metabolic equivalents; vBMD, volumetric bone mineral density.

*Corresponding author: L. A. Bazzano, fax +1 504988 1568, email lbazzano@tulane.edu 
In ecological studies of animal protein intake and rates of hip fracture, the highest rates were observed in Western countries where dietary protein intake, particularly from animal sources, is also highest ${ }^{(8,9)}$. Consumption of animal proteins, high in acidifying amino acids such as cysteine and methionine, could potentially increase the risk of bone loss ${ }^{(10,11)}$. A few large prospective cohort studies have also associated higher protein intake (particularly from animal sources) with higher incidence of hip fracture ${ }^{(12-14)}$, while others have found the opposite association $^{(15-17)}$, and one found no association ${ }^{(18)}$. Aside from differences in measurement methods, this inconsistency may be due in part to racial/ethnic differences. Recent studies have suggested that the association between protein intake and bone mineral density (BMD) may differ by race and/or ethnicity ${ }^{(19-24)}$, which could reflect differences in dietary patterns and sources of protein.

Diagnosis of osteoporosis and clinical guidelines for its management are often based on BMD of the lumbar spine, total hip or femoral neck ${ }^{(25,26)}$. Measures of volumetric or three-dimensional BMD can be obtained from computed tomography (CT) scans, while traditional dual-energy X-ray absorptiometry scans provide two-dimensional areal BMD. Unlike dual-energy X-ray absorptiometry scans, quantitative CT scans can also provide separate measures for cortical and trabecular compartments of bone, aspects of geometry and architecture that may be related to fracture $\operatorname{risk}^{(25,27,28)}$.

In the present study, we examined the association between dietary protein and volumetric bone mineral density (vBMD) of the lumbar spine obtained from quantitative CT, specifically the average of lumbar vBMD in the second through the fourth lumbar vertebrae (primary outcome) and vBMD $Z$-score generated by subtracting the mean and dividing the difference by the standard deviation for each observation based on sex- and race/ethnicity-specific distributions (secondary outcome) among participants of the Multi-Ethnic Study of Atherosclerosis (MESA). We also evaluated whether relationships differed by source of protein intake (animal or vegetable), sex and race/ethnicity (non-Hispanic white, Chinese-American, African-American and Hispanic-American).

\section{Methods}

\section{Participants}

The methods of the MESA study have been described in detail elsewhere $^{(29)}$. In brief, the MESA cohort was initially examined between July 2000 and August 2002 (baseline). Participants were recruited from six US communities. The study population consisted of 6814 men and women aged $45-84$ years who were free of CVD at their baseline examination. Approximately $38 \%$ of the cohort was non-Hispanic white, $11 \%$ Chinese-American, $28 \%$ African-American and 23\% Hispanic-American. We used data from a subsample of MESA participants who participated in the MESA Abdominal Aortic Calcium Study. Between August 2002 and September 2005, participants were recruited to the MESA Abdominal Aortic Calcium Study during their follow-up visits in the following five MESA communities: Chicago, IL; Forsyth County, NC; Los Angeles County, CA;
New York, NY; St Paul, MN. In total, 1967 men and women who participated in the MESA Abdominal Aortic Calcium Study completed CT scanning. Of those, data from fourteen participants were excluded because they lacked data on bone density of the second through fourth lumbar vertebrae; 235 participants who reported consuming $>25104 \mathrm{~kJ} / \mathrm{d}$ or $<2510 \mathrm{~kJ} / \mathrm{d}$ were excluded; and an additional sixty were excluded because they provided implausible data on physical activity $(>24 \mathrm{~h} / \mathrm{d})$. After these exclusions, data from 1658 participants were included in the present analysis. The MESA study was conducted according to the guidelines of the Declaration of Helsinki, and all MESA procedures were approved by the institutional review boards of participating institutions. Written informed consent was obtained from all MESA participants during primary data collection. This secondary data analysis was approved by the institutional review board of Tulane University.

\section{Bone density measurement}

The measurement of vBMD using CT scans of the abdomen in MESA has been reported in a previous study ${ }^{(30)}$. Scanning was performed using an electron-beam CT scanner (Chicago, IL; New York, NY; and Los Angeles, CA, USA; Imatron C-150; General Electric Medical Systems) or a multi-detector CT system that utilised helical scanning with reconstruction in $5 \mathrm{~mm}$ thick cuts and $350 \mathrm{~mm}$ field of view (New York, NY; Forsyth County, NC; and St Paul, MN field centres, USA; Siemens, Inc., General Electric Medical system). During the scanning, CT parameters were directly converted to equivalent vBMD in $\mathrm{mg} / \mathrm{cm}^{3(31)}$. Using the Image Analysis QCT 3D PLUS software program (Image Analysis), vBMD was measured in a virtual $10 \mathrm{~mm}$-thick slice from each vertebral bone (L2-L4) using software-directed, automated placement of the region of interest in the anterior one-half to one-third of the vertebral body, where it encompassed a large area of trabecular or cancellous bone, excluded cortical bone, and excluded the basivertebral plexus. A trained reader adjusted placement of the reading region and excluded vertebrae if fractures, metastatic lesions, osteophytes or benign focal lesions were detected. All scans were brightness adjusted with a standard phantom. The scans were read by a blinded, experienced reader at the MESA CT reading centre. Previous studies have demonstrated the comparability and reproducibility of image data collected using these scanners ${ }^{(32,33)}$

\section{Dietary assessment}

All participants were asked to complete a 120-item FFQ at baseline, on average 3 years before the CT scan, to assess their usual intake of specific foods and beverages over the past year. The FFQ utilised the Block format and was patterned after the FFQ used in the Insulin Resistance Atherosclerosis Study, which has been validated among non-Hispanic white, African-American and HispanicAmerican, and modified to include Chinese foods ${ }^{(34,35)}$. For each food item, the consumption frequency (times/d, week or month) and serving size (small, medium or large) were 
recorded. Frequency options included nine responses ranging from 'rare or never' to ' $\geq 2$ times/d' for each food item ${ }^{(35)}$. Related line items were combined to form forty-seven different food groups ${ }^{(35)}$. Daily macronutrient and micronutrient intakes were estimated by multiplying frequency and serving size (age- and sex-specific and portion-size gram weights) for each food/beverage consumed by the nutrient content of that food or beverage (Nutrition Data Systems for Research; University of Minnesota; Minneapolis). Dietary protein intake, including total, animal and vegetable protein, was calculated in $\mathrm{g}$ and also as a percentage of total energy intake (\% energy). In studies examining the reproducibility of a single FFQ, correlations ranged between 0.5 and 0.7 for nutrient intakes measured at intervals of 1 to 10 years ${ }^{(36)}$.

\section{Additional variables}

Baseline data collection also included a medical history, standardised medical examination, laboratory tests and anthropometric measurements. Age, sex, race/ethnicity, age at menopause, educational level, current cigarette smoking and current use of hormone replacement therapy were collected using interviewer and self-administered questionnaires. We calculated BMI at baseline as weight $(\mathrm{kg})$ divided by height $(\mathrm{m})$ squared. Physical activity was assessed as metabolic equivalents (MET), using a semi-quantitative questionnaire adapted from the Cross-Cultural Activity Participation Study ${ }^{(37)}$. Leisure-time physical activity was calculated as the sum of min/week of moderate to vigorous activities (walking, sports, dance and conditioning) multiplied by the activity's individual MET value. Likewise, a sedentariness score was calculated as the sum of MET-min/week of sitting, reclining, knitting, sewing, reading, driving a car and watching television. Estimated glomerular filtration rate was calculated using the CKD-EPI (Chronic Kidney Disease Epidemiology Collaboration) equation ${ }^{(38)}$.

\section{Statistical analysis}

For each baseline characteristic, the mean value or corresponding percent of study participants was calculated by quartile of total protein intake as a percentage of total energy. The statistical significance of differences among quartiles was examined using $\chi^{2}$ tests for categorical variables and generalised linear regression analyses for continuous variables.

There was evidence that the association between dietary protein intake and BMD differed by race/ethnicity and $\operatorname{sex}(P$ for interaction $=0.03$ for protein (as \% energy) and $P$ for interaction $=0.02$ for protein (in g)). Thus, we categorised participants into groups based on sex- and race/ ethnicity-specific quartiles of dietary protein intake for analyses.

We used substitution modelling to investigate the association of 'substituting' an energy equivalent amount of dietary protein (total, animal and/or vegetable protein) for fat with lumbar spine vBMD by including dietary protein and dietary carbohydrate intakes in the same multivariable model with total energy intake. Tests for linear trend were conducted for adjusted means of vBMD across the quartiles of energy intake from dietary protein (for total, animal and vegetable protein).
Adjusted mean vBMD was computed by quartile of sex- and race/ethnicity-specific protein intake using linear regression models. In multivariable-adjusted models, a variety of potential confounders including age (years), BMI $\left(\mathrm{kg} / \mathrm{m}^{2}\right)$, physical activity (MET-min/week), sedentariness score (MET-min/week), current smoking (yes/no), educational level ( $\leq$ high school $v$. $\geq$ college), hormone replacement therapy use, age at menopause (years), and intakes of total energy $(\mathrm{kJ} / \mathrm{d})$, dietary carbohydrate (\% energy), Ca (mg), $\mathrm{P}(\mathrm{mg}), \mathrm{Mg}(\mathrm{mg})$ and alcohol $(\mathrm{g} / \mathrm{d})$ were included. We examined the influence of renal function by including estimated glomerular filtration rate, and generated a propensity score to represent $\mathrm{Ca}, \mathrm{Mg}$ and $\mathrm{P}$ intakes as a single covariate. In order to limit the influence of heteroscedasticity or potential outliers in the present analysis, robust regression models were employed to examine the impact of quartile of dietary protein intake (as \% energy), one standard deviation changes in protein intake (as \% energy), and absolute protein intake (in g) on vBMD $Z$-score among the different racial/ethnic groups. We tested for interactions between dietary protein intake and hormone replacement therapy as well as $\mathrm{Ca}$ intake. All $P$ values were two-sided, and statistical significance was defined as $P<0 \cdot 05$. Multiple comparisons were controlled by using the false discovery rate $\operatorname{method}^{(39)}$. We used SAS (version 9.2; SAS Institute, Inc.) for all analyses.

\section{Results}

The present analysis included 1658 participants, of whom 801 or $48 \%$ were women. Of these women, 317 (39.6\%) were non-Hispanic white, 104 (13.0\%) were Chinese-American, $173(21.6 \%)$ were African-American and 207 (25.8\%) were Hispanic-American. Of men, 375 (43.8\%), 126 (14.7\%), $133(15 \cdot 5 \%)$ and $223(26 \cdot 0 \%)$ were non-Hispanic white, Chinese-American, African-American and Hispanic-American, respectively. The mean age among women was 63 years and that among men was 62 years. Baseline characteristics are presented by quartile of dietary intake of total protein in Table 1. Those in the highest quartile of protein intake were slightly younger, more often female and less likely to smoke cigarettes. Non-Hispanic whites and African-Americans tended to consume less protein, while roughly half of Chinese were in the highest quartile of protein intake. The amount of vegetable protein and fat intakes (as \% energy) was consistent across the quartiles, but intake of animal protein was higher and carbohydrate was lower among those with higher total protein intake.

We identified a significant interaction by which race/ ethnicity modified the effect of dietary protein on vBMD; therefore, race/ethnicity-specific quartiles of protein intake (\% energy) were used to assess the association between vBMD and protein intake in men and women separately. These quartiles are presented in Table 2.

No significant relationships were observed among men of any race/ethnicity (Table 3). For women, there was no significant interaction between dietary protein and hormone replacement therapy use or $\mathrm{Ca}$ intake, in association with lumbar spine vBMD. Non-Hispanic white women with higher vegetable protein intake had a higher mean vBMD 
Table 1. Characteristics by quartile (Q) of dietary intake of total protein in 1658 participants of the Multi-Ethnic Study of Atherosclerosis (MESA) 2000-5

(Mean values and standard deviations or percentages)

\begin{tabular}{|c|c|c|c|c|c|c|c|c|c|}
\hline \multirow[b]{3}{*}{ Variables } & \multicolumn{9}{|c|}{ Quartile of total protein intake (\% of energy intake) ${ }^{*}$} \\
\hline & \multicolumn{2}{|c|}{ Q1 } & \multicolumn{2}{|c|}{ Q2 } & \multicolumn{2}{|c|}{ Q3 } & \multicolumn{2}{|c|}{ Q4 } & \multirow{2}{*}{$\begin{array}{l}P \text { for } \\
\text { trend }\end{array}$} \\
\hline & Mean & SD & Mean & SD & Mean & SD & Mean & SD & \\
\hline Participants $(n)$ & 414 & & 415 & & 413 & & 416 & & \\
\hline Age (years) & $62 \cdot 2$ & 9.9 & 63.4 & 10 & 61.5 & $10 \cdot 0$ & $62 \cdot 1$ & $9 \cdot 3$ & 0.05 \\
\hline Men $(\%)$ & $54 \cdot 2$ & & 55.5 & & $50 \cdot 4$ & & $46 \cdot 6$ & & 0.03 \\
\hline \multicolumn{10}{|l|}{ Race/ethnicity (\%) } \\
\hline White & $46 \cdot 1$ & & $45 \cdot 2$ & & $39 \cdot 2$ & & $36 \cdot 5$ & & 0.01 \\
\hline Chinese & $6 \cdot 1$ & & 8.6 & & $15 \cdot 3$ & & $26 \cdot 0$ & & $<0.001$ \\
\hline Black & $21 \cdot 7$ & & $21 \cdot 3$ & & $17 \cdot 3$ & & $12 \cdot 5$ & & 0.002 \\
\hline Hispanic & $26 \cdot 1$ & & 24.9 & & $28 \cdot 2$ & & $25 \cdot 0$ & & 0.59 \\
\hline College & 64.4 & & $65 \cdot 3$ & & $62 \cdot 8$ & & $66 \cdot 6$ & & 0.48 \\
\hline BMI $\left(\mathrm{kg} / \mathrm{m}^{2}\right)$ & $28 \cdot 2$ & $5 \cdot 2$ & 28.2 & 5.0 & $27 \cdot 9$ & 5.0 & $27 \cdot 8$ & $5 \cdot 2$ & 0.51 \\
\hline Age at menopause (years) & $47 \cdot 0$ & $6 \cdot 9$ & $48 \cdot 4$ & $6 \cdot 4$ & $47 \cdot 7$ & $6 \cdot 3$ & 48.4 & $5 \cdot 8$ & 0.08 \\
\hline Physical activity (MET-min/week) & 5249 & 3940 & 5399 & 3594 & 5101 & 3441 & 4989 & 3616 & 0.40 \\
\hline $\begin{array}{l}\text { Sedentariness score } \\
\text { (MET-min/week) }\end{array}$ & 2400 & 1302 & 2476 & 1225 & 2297 & 1258 & 2329 & 1201 & 0.17 \\
\hline \multicolumn{10}{|l|}{ Baseline diet } \\
\hline Total energy (kJ) & 6945 & 3335 & 6770 & 3293 & 6460 & 3025 & 5966 & 2900 & $<0.001$ \\
\hline Carbohydrate (\%) & $55 \cdot 8$ & $10 \cdot 4$ & 53.5 & $7 \cdot 8$ & $52 \cdot 1$ & $7 \cdot 5$ & $49 \cdot 6$ & $8 \cdot 2$ & $<0.001$ \\
\hline Fat (\%) & $30 \cdot 8$ & $7 \cdot 9$ & 31.5 & $6 \cdot 6$ & 31.4 & $6 \cdot 6$ & $30 \cdot 7$ & 6.9 & 0.23 \\
\hline Total protein $(\mathrm{g})$ & $49 \cdot 6$ & 24.5 & $59 \cdot 2$ & $28 \cdot 7$ & 64.5 & $30 \cdot 4$ & 71.5 & $35 \cdot 1$ & $<0.001$ \\
\hline Animal protein $(\mathrm{g})$ & $28 \cdot 2$ & $17 \cdot 8$ & 36.6 & $20 \cdot 1$ & $42 \cdot 4$ & $22 \cdot 3$ & $50 \cdot 6$ & $28 \cdot 1$ & $<0.001$ \\
\hline Vegetable protein (g) & $20 \cdot 7$ & $10 \cdot 3$ & 21.8 & $10 \cdot 9$ & 21.9 & $10 \cdot 7$ & $20 \cdot 5$ & $11 \cdot 2$ & 0.14 \\
\hline $\mathrm{Ca}(\mathrm{mg})$ & 589 & 379 & 729 & 466 & 794 & 542 & 886 & 659 & $<0.001$ \\
\hline $\mathrm{Mg}(\mathrm{mg})$ & 245 & 110 & 269 & 124 & 272 & 123 & 283 & 150 & $<0.001$ \\
\hline$P(\mathrm{mg})$ & 904 & 462 & 1050 & 549 & 1109 & 579 & 1195 & 659 & $<0.001$ \\
\hline Alcohol (g) & $10 \cdot 4$ & 23.3 & $6 \cdot 3$ & $13 \cdot 2$ & $3 \cdot 87$ & $6 \cdot 7$ & 2.9 & 5.3 & $<0.001$ \\
\hline Cold cereal (servings/d) $\dagger$ & 0.26 & 0.33 & 0.31 & 0.38 & 0.25 & 0.34 & 0.22 & 0.32 & 0.001 \\
\hline Multivitamin use (\%) & 63.4 & & $61 \cdot 4$ & & $59 \cdot 9$ & & $65 \cdot 9$ & & 0.32 \\
\hline Whole grain (servings/d) & 0.55 & 0.55 & 0.63 & 0.58 & 0.59 & 0.57 & 0.52 & 0.52 & 0.03 \\
\hline $\operatorname{vBMD}\left(\mathrm{mg} / \mathrm{cm}^{3}\right) \ddagger$ & 115 & 40 & 115 & 38 & 116 & 42 & 112 & 39 & 0.88 \\
\hline Current smoker (\%) & $15 \cdot 9$ & & $12 \cdot 7$ & & $10 \cdot 7$ & & $9 \cdot 3$ & & 0.03 \\
\hline HRT current use (\%)§ & $37 \cdot 0$ & & $37 \cdot 3$ & & $32 \cdot 1$ & & 31.9 & & 0.53 \\
\hline
\end{tabular}

MET, metabolic equivalents; vBMD, volumetric bone mineral density; HRT, hormone replacement therapy.

${ }^{*}$ The ranges for the quartiles $1-4$ of dietary total protein intake were $6 \cdot 1-13 \cdot 6,13 \cdot 7-15 \cdot 7,15 \cdot 8-17 \cdot 9$ and $18 \cdot 0-33 \cdot 5$, respectively.

† Cold cereal is a part of whole grain.

$\ddagger$ Trabecular vBMD of Lumbar L2-4 vertebrae.

$\S$ Female only.

( $P$ for trend $=0.03$ ), after adjustment for age, BMI, physical activity, sedentariness score, current smoking, educational level, hormone therapy use, age at menopause, and intakes of total energy, dietary carbohydrate, animal protein, $\mathrm{Ca}, \mathrm{P}$, $\mathrm{Mg}$ and alcohol (Table 3). The associations with regard to total and animal protein intakes were not significant among non-Hispanic white women.

We further examined whether the associations observed among women could be due to outliers or heterogeneity by employing robust regression methods. These results were consistent with the adjusted robust regression analyses examining the association between protein intake and vBMD $Z$-score (Table 4). Compared with the lowest quartile of vegetable protein intake, non-Hispanic white women in the highest quartile had significantly greater lumbar spine vBMD $Z$-scores after adjusting for potential confounders. Moreover, this result was also consistent when continuous dietary vegetable protein intake was used in robust regression models; a greater intake of vegetable protein was significantly associated with a higher vBMD (for each $1 \%$ greater protein intake lumbar spine vBMD $Z$-score was $3.87 \%$ greater,
$P=0 \cdot 01$ ) among non-Hispanic white women. In contrast, there were no consistently significant associations between dietary protein (total, animal or vegetable protein) intake and vBMD in other sex and racial/ethnic groups. For example, Hispanic-American women with higher animal protein intake had a higher mean vBMD ( $P$ for trend $=0.04$ ), and ChineseAmerican women with higher animal protein intake had a lower mean vBMD ( $P$ for trend $=0 \cdot 02$ ) (Table 3 ). These associations were no longer significant in robust regression analyses, regardless of whether the categorical (Table 4) or continuous (data not shown) dietary intake of animal protein was used in the models. Additional models without substitution of fat for protein or the exchange of protein for carbohydrate did not alter the results (data not shown). Modelling absolute protein intakes as one standard deviation difference (in g) was not associated with lumbar spine vBMD (see online supplementary Appendix Table S1), and there was no alteration of the results after further adjustment for estimated glomerular filtration rate or propensity score representing $\mathrm{Ca}, \mathrm{P}$ and $\mathrm{Mg}$ (see online supplementary Appendix Tables S2-S8). 
Table 2. Medians of dietary protein intake by type of protein, race/ethnicity and sex (Medians and interquartile ranges (IQR))

\begin{tabular}{|c|c|c|c|c|c|c|c|c|}
\hline & \multicolumn{8}{|c|}{ Quartile of protein intake (\% of energy intake) } \\
\hline & \multicolumn{2}{|c|}{ Q1 } & \multicolumn{2}{|c|}{ Q2 } & \multicolumn{2}{|c|}{ Q3 } & \multicolumn{2}{|c|}{ Q4 } \\
\hline & Median & IQR & Median & IQR & Median & IQR & Median & IQR \\
\hline \multicolumn{9}{|l|}{ Women } \\
\hline \multicolumn{9}{|c|}{ White ( $n$ 317) } \\
\hline TP & $12 \cdot 6$ & $8 \cdot 3-13 \cdot 5$ & $14 \cdot 6$ & $13 \cdot 6-15 \cdot 7$ & $16 \cdot 8$ & $15 \cdot 8-17 \cdot 9$ & $19 \cdot 8$ & $18 \cdot 0-30 \cdot 7$ \\
\hline AP & $7 \cdot 0$ & $3 \cdot 0-8 \cdot 0$ & 8.9 & $8 \cdot 1-10 \cdot 1$ & $11 \cdot 1$ & $10 \cdot 2-12 \cdot 4$ & $14 \cdot 7$ & $12 \cdot 5-27 \cdot 9$ \\
\hline VP & $4 \cdot 0$ & $1 \cdot 8-4 \cdot 4$ & $4 \cdot 8$ & $4 \cdot 5-5 \cdot 2$ & $5 \cdot 5$ & $5 \cdot 3-6 \cdot 1$ & $7 \cdot 0$ & $6 \cdot 2-14.9$ \\
\hline \multicolumn{9}{|c|}{ Chinese ( $n$ 104) } \\
\hline TP & $14 \cdot 6$ & $10 \cdot 4-15 \cdot 9$ & $16 \cdot 9$ & $16 \cdot 0-18 \cdot 1$ & $19 \cdot 3$ & $18 \cdot 2-20 \cdot 6$ & $22 \cdot 0$ & $20 \cdot 7-30 \cdot 1$ \\
\hline AP & $6 \cdot 4$ & $1 \cdot 2-8 \cdot 4$ & 9.9 & $8.5-11.3$ & $12 \cdot 6$ & $11 \cdot 4-13 \cdot 4$ & $15 \cdot 3$ & $13 \cdot 5-25 \cdot 8$ \\
\hline VP & $5 \cdot 6$ & $3 \cdot 4-6 \cdot 1$ & $6 \cdot 6$ & $6 \cdot 2-7 \cdot 1$ & 7.4 & $7 \cdot 2-7.9$ & $8 \cdot 6$ & $8 \cdot 0-13 \cdot 6$ \\
\hline \multicolumn{9}{|c|}{ Black ( $n$ 173) } \\
\hline TP & $12 \cdot 0$ & $6 \cdot 0-13 \cdot 4$ & $14 \cdot 6$ & $13 \cdot 5-15 \cdot 6$ & $16 \cdot 5$ & $15 \cdot 7-17 \cdot 3$ & $18 \cdot 9$ & $17.4-24.5$ \\
\hline AP & 6.5 & $2 \cdot 5-8 \cdot 0$ & $9 \cdot 4$ & $8 \cdot 1-10 \cdot 1$ & $11 \cdot 2$ & $10 \cdot 2-11 \cdot 9$ & $13 \cdot 4$ & $12 \cdot 0-19 \cdot 7$ \\
\hline VP & 3.6 & $2 \cdot 5-4.3$ & 4.8 & $4 \cdot 4-5 \cdot 2$ & 5.6 & $5 \cdot 3-6 \cdot 3$ & $6 \cdot 9$ & $6 \cdot 4-12 \cdot 7$ \\
\hline \multicolumn{9}{|c|}{ Hispanics ( $n$ 207) } \\
\hline TP & $12 \cdot 3$ & $8 \cdot 0-13 \cdot 5$ & 14.9 & $13 \cdot 6-15 \cdot 8$ & $16 \cdot 9$ & $15 \cdot 9-18 \cdot 1$ & $19 \cdot 7$ & $18 \cdot 2-25 \cdot 0$ \\
\hline AP & 5.9 & $2 \cdot 2-7 \cdot 7$ & 9.0 & $7 \cdot 8-9 \cdot 8$ & $10 \cdot 7$ & $9 \cdot 9-12 \cdot 4$ & $14 \cdot 6$ & $12 \cdot 5-20 \cdot 9$ \\
\hline VP & 4.0 & $2 \cdot 1-4 \cdot 6$ & $5 \cdot 2$ & $4 \cdot 7-5 \cdot 6$ & $6 \cdot 1$ & $5 \cdot 7-6 \cdot 8$ & $7 \cdot 7$ & $6 \cdot 9-9 \cdot 8$ \\
\hline \multicolumn{9}{|l|}{ Men } \\
\hline \multicolumn{9}{|c|}{ White ( $n$ 375) } \\
\hline TP & 11.9 & $6 \cdot 4-13 \cdot 2$ & $14 \cdot 0$ & $13 \cdot 3-15 \cdot 0$ & $16 \cdot 0$ & $15 \cdot 1-16 \cdot 8$ & $18 \cdot 6$ & $16 \cdot 9-33 \cdot 6$ \\
\hline AP & $6 \cdot 2$ & $2 \cdot 1-7 \cdot 6$ & $8 \cdot 6$ & $7 \cdot 7-9 \cdot 3$ & $10 \cdot 6$ & $9 \cdot 4-11 \cdot 7$ & $13 \cdot 7$ & $11 \cdot 8-31 \cdot 2$ \\
\hline VP & 3.7 & $1 \cdot 5-4.2$ & $4 \cdot 6$ & $4 \cdot 3-4.9$ & 5.4 & $5 \cdot 0-5 \cdot 8$ & $6 \cdot 6$ & $5 \cdot 9-10 \cdot 4$ \\
\hline \multicolumn{9}{|c|}{ Chinese ( $n$ 126) } \\
\hline TP & $13 \cdot 4$ & $9 \cdot 3-15 \cdot 4$ & $16 \cdot 2$ & $15 \cdot 5-17 \cdot 1$ & $18 \cdot 2$ & $17 \cdot 2-18 \cdot 9$ & $20 \cdot 4$ & $19 \cdot 0-28 \cdot 7$ \\
\hline AP & $6 \cdot 3$ & $2.9-7.9$ & 9.5 & $8.0-10 \cdot 2$ & 11.0 & $10 \cdot 3-12 \cdot 5$ & 14.5 & $12 \cdot 6-23 \cdot 5$ \\
\hline VP & $5 \cdot 1$ & $2 \cdot 7-5.9$ & $6 \cdot 3$ & $5 \cdot 9-6 \cdot 6$ & $7 \cdot 2$ & $6 \cdot 7-7 \cdot 7$ & $8 \cdot 4$ & $7 \cdot 8-15 \cdot 4$ \\
\hline \multicolumn{9}{|c|}{ Black (n 133) } \\
\hline TP & 11.6 & $8 \cdot 3-12 \cdot 6$ & $13 \cdot 9$ & $12 \cdot 7-14.9$ & $15 \cdot 3$ & $15 \cdot 0-16 \cdot 6$ & 18.5 & $16 \cdot 7-22 \cdot 8$ \\
\hline AP & $6 \cdot 4$ & $0.1-7.4$ & $8 \cdot 2$ & $7 \cdot 5-9 \cdot 2$ & $10 \cdot 2$ & $9 \cdot 3-11 \cdot 1$ & $13 \cdot 5$ & $11 \cdot 2-18 \cdot 6$ \\
\hline VP & 3.6 & $1 \cdot 4-4 \cdot 1$ & 4.6 & $4.2-4.9$ & 5.5 & $5 \cdot 0-5 \cdot 9$ & $7 \cdot 2$ & $6.0-11.9$ \\
\hline \multicolumn{9}{|c|}{ Hispanics (n 223) } \\
\hline TP & $12 \cdot 3$ & $7 \cdot 1-13 \cdot 6$ & 14.5 & $13 \cdot 7-15 \cdot 6$ & $16 \cdot 6$ & $15 \cdot 7-17 \cdot 6$ & $19 \cdot 0$ & $17 \cdot 7-24 \cdot 3$ \\
\hline AP & $6 \cdot 2$ & $2.6-7.5$ & 8.7 & $7 \cdot 6-9 \cdot 7$ & $10 \cdot 4$ & $9 \cdot 8-11 \cdot 7$ & $13 \cdot 5$ & $11 \cdot 8-19.9$ \\
\hline VP & 4.0 & $1.4-4.4$ & $5 \cdot 0$ & $4 \cdot 5-5 \cdot 4$ & 5.9 & $5 \cdot 5-6 \cdot 4$ & 7.5 & $6 \cdot 5-18 \cdot 7$ \\
\hline
\end{tabular}

$\mathrm{Q}$, quartile; TP, total protein; AP, animal protein; VP, vegetable protein.

We also made adjustment for multiple comparisons to limit the potential for false-positive findings. After adjustment for multiple comparisons, the observed associations did not reach conventional levels of significance.

\section{Discussion}

In the present multi-ethnic cohort of middle-aged men and women from the MESA cohort, sex and race/ethnicity jointly modified the association of dietary protein with lumbar spine trabecular vBMD. No detrimental effects of high-protein diets on vBMD were observed. In contrast, the most consistent association was that of a higher vegetable protein intake substituting for fat with a greater lumbar spine vBMD among non-Hispanic white women, independent of age, BMI, smoking, physical activity, postmenopausal hormone use, age at menopause, and intakes of total energy, carbohydrate, Ca, P, $\mathrm{Mg}$ and alcohol. However, this association was not statistically significant after adjustment for multiple comparisons.

It is still unknown whether the relationship between protein intake and bone health is modified by $\mathrm{Ca}$ intake. We did not observe a significant interaction between dietary Ca and protein intake in our sample. In a randomised controlled trial among 342 healthy men and women (mean age 71 years; mean total protein intake $79 \cdot 1 \mathrm{~g} / \mathrm{d}$ ), Dawson-Hughes \& Harris ${ }^{(40)}$ reported that the association of total protein intake with a 3-year change in total and femoral neck BMD was significantly different between groups with and without $\mathrm{Ca}$ /vitamin $\mathrm{D}$ supplementation, but not for lumbar spine BMD. A Ca balance study among fifteen postmenopausal, mostly white, women (mean age 61 years) showed that urinary Ca loss or indicators of bone metabolism did not differ between low-protein (mean total protein intake $68 \mathrm{~g} / \mathrm{d}$ ) and high-protein (mean total protein intake $117 \mathrm{~g} / \mathrm{d}$ ) diets ${ }^{(41)}$. In contrast, a prospective cohort study among 960 older men and women has shown that increasing protein consumption appeared to be more beneficial to women with lower Ca intakes (mean age 71 years) ${ }^{(17)}$.

Our findings with regard to total and animal protein intakes are consistent with several studies that examined this association among postmenopausal women ${ }^{(10,22,42)}$. In contrast, Teegarden et $a l .{ }^{(43)}$ and Quintas et al. ${ }^{(44)}$ found a positive association of total protein intake with lumbar spine BMD 
Table 3. Adjusted mean lumbar spine trabecular volumetric bone mineral density (in $\mathrm{mg} / \mathrm{cm}^{3}$ ) by race/ethnicity and protein intake at baseline (Adjusted mean values and $95 \%$ confidence intervals, $n$ 1658)

\begin{tabular}{|c|c|c|c|c|c|c|c|c|c|}
\hline & \multicolumn{9}{|c|}{ Quartile of protein intake (\% of energy intake) ${ }^{*}$} \\
\hline & \multicolumn{2}{|c|}{ Q1 } & \multicolumn{2}{|c|}{ Q2 } & \multicolumn{2}{|c|}{ Q3 } & \multicolumn{2}{|c|}{ Q4 } & \multirow[b]{2}{*}{$P$ for treno } \\
\hline & Adjusted mean & $95 \% \mathrm{Cl}$ & Adjusted mean & $95 \% \mathrm{Cl}$ & Adjusted mean & $95 \% \mathrm{Cl}$ & Adjusted mean & $95 \% \mathrm{Cl}$ & \\
\hline \multicolumn{10}{|l|}{ Women } \\
\hline \multicolumn{10}{|l|}{ White } \\
\hline TP & $102 \cdot 0$ & $93 \cdot 6,110 \cdot 4$ & 99.0 & $91 \cdot 2,106 \cdot 8$ & $96 \cdot 8$ & $89 \cdot 3,104 \cdot 4$ & $98 \cdot 3$ & $89 \cdot 4,107 \cdot 2$ & 0.57 \\
\hline APß & $101 \cdot 3$ & $92 \cdot 8,109 \cdot 7$ & $100 \cdot 8$ & $93 \cdot 1,108 \cdot 5$ & 97.5 & $89 \cdot 9,105 \cdot 1$ & $97 \cdot 3$ & $87 \cdot 7,106 \cdot 8$ & 0.49 \\
\hline VP\| & 93.7 & $85 \cdot 2,102 \cdot 2$ & 97.6 & $90 \cdot 1,105 \cdot 2$ & 99.0 & $91 \cdot 3,106 \cdot 7$ & 107.4 & $98 \cdot 6,116 \cdot 2$ & 0.03 \\
\hline \multicolumn{10}{|c|}{ Chinese } \\
\hline TP & $118 \cdot 8$ & $86 \cdot 7,150 \cdot 9$ & 127.9 & $93 \cdot 2,162 \cdot 6$ & $116 \cdot 4$ & $82 \cdot 0,150 \cdot 8$ & $109 \cdot 3$ & $67 \cdot 7,150 \cdot 8$ & 0.38 \\
\hline AP & 136.5 & $102 \cdot 5,170 \cdot 4$ & $127 \cdot 0$ & $94 \cdot 7,159 \cdot 3$ & 111.3 & $77 \cdot 5,145 \cdot 0$ & 107.5 & $68 \cdot 0,146 \cdot 9$ & 0.02 \\
\hline VP & 122.4 & $89.6,155.2$ & $126 \cdot 6$ & $93.4,159.8$ & 136.5 & $99.9,173 \cdot 2$ & 139.4 & $102 \cdot 5,176 \cdot 2$ & $0 \cdot 10$ \\
\hline \multicolumn{10}{|l|}{ Black } \\
\hline TP & $119 \cdot 0$ & $103 \cdot 1,134.9$ & $146 \cdot 4$ & $132.8,159.9$ & $129 \cdot 1$ & $116 \cdot 6,141 \cdot 6$ & $137 \cdot 0$ & $121 \cdot 7,152 \cdot 4$ & 0.30 \\
\hline AP & $120 \cdot 8$ & $106 \cdot 2,138 \cdot 1$ & 136.5 & $122 \cdot 3,150 \cdot 6$ & $140 \cdot 1$ & $126 \cdot 9,153 \cdot 4$ & 134.9 & $117 \cdot 3,152 \cdot 4$ & 0.21 \\
\hline VP & $142 \cdot 0$ & $127 \cdot 3,156 \cdot 8$ & $131 \cdot 6$ & $117 \cdot 5,145 \cdot 7$ & $129 \cdot 1$ & $115 \cdot 2,143 \cdot 0$ & $125 \cdot 8$ & $110 \cdot 3,141 \cdot 4$ & 0.17 \\
\hline \multicolumn{10}{|c|}{ Hispanics } \\
\hline TP & $105 \cdot 2$ & $90 \cdot 7,119 \cdot 7$ & $107 \cdot 2$ & $95 \cdot 7,118 \cdot 7$ & $110 \cdot 6$ & $98 \cdot 5,122 \cdot 8$ & $115 \cdot 7$ & $101 \cdot 8,129 \cdot 7$ & 0.25 \\
\hline AP & $100 \cdot 0$ & $86 \cdot 3,113 \cdot 8$ & $105 \cdot 7$ & $93 \cdot 6,117 \cdot 8$ & $112 \cdot 6$ & $101 \cdot 5,123 \cdot 8$ & $120 \cdot 5$ & $105 \cdot 2,135 \cdot 8$ & 0.04 \\
\hline VP & $102 \cdot 6$ & $89 \cdot 0,116 \cdot 1$ & 113.9 & $101 \cdot 6,126 \cdot 3$ & $106 \cdot 1$ & $94 \cdot 4,117 \cdot 8$ & $112 \cdot 8$ & $100 \cdot 5,125 \cdot 2$ & 0.31 \\
\hline \multicolumn{10}{|l|}{ Men } \\
\hline \multicolumn{10}{|l|}{ White } \\
\hline TP & $112 \cdot 2$ & $103 \cdot 4,120 \cdot 8$ & $113 \cdot 7$ & $106 \cdot 3,121 \cdot 2$ & 111.5 & $104 \cdot 1,118 \cdot 8$ & $107 \cdot 1$ & $98 \cdot 2,116 \cdot 0$ & 0.40 \\
\hline AP & 108.0 & $99 \cdot 3,116 \cdot 7$ & $115 \cdot 2$ & $107 \cdot 6,122 \cdot 8$ & $110 \cdot 7$ & $103 \cdot 3,118 \cdot 1$ & $110 \cdot 3$ & $100 \cdot 8,119 \cdot 7$ & 0.89 \\
\hline VP & $106 \cdot 8$ & $98 \cdot 9,114 \cdot 7$ & $119 \cdot 1$ & $111 \cdot 6,126 \cdot 5$ & 114.9 & $107 \cdot 7,122 \cdot 0$ & $105 \cdot 0$ & $97 \cdot 1,112 \cdot 9$ & 0.53 \\
\hline \multicolumn{10}{|c|}{ Chinese } \\
\hline TP & 103.9 & $88 \cdot 6,119 \cdot 1$ & $107 \cdot 0$ & $95 \cdot 0,119 \cdot 0$ & $121 \cdot 3$ & $109 \cdot 7,133.0$ & $118 \cdot 8$ & $103 \cdot 3,134 \cdot 3$ & 0.13 \\
\hline AP & 102.7 & $86 \cdot 3,119 \cdot 1$ & $117 \cdot 1$ & $105.0,129.2$ & 113.7 & $101 \cdot 6,125 \cdot 7$ & 115.9 & $99.5,132.2$ & 0.35 \\
\hline VP & 112.9 & $98 \cdot 3,127 \cdot 6$ & 107.5 & $95.4,119.5$ & $120 \cdot 1$ & $108 \cdot 6,131 \cdot 6$ & $109 \cdot 6$ & $94 \cdot 8,124 \cdot 4$ & 0.77 \\
\hline \multicolumn{10}{|l|}{ Black } \\
\hline TP & 131.0 & $110 \cdot 6,151 \cdot 5$ & 142.9 & $127 \cdot 1,158 \cdot 7$ & 141.6 & $126 \cdot 7,156 \cdot 6$ & 158.6 & $138.9,178.2$ & 0.11 \\
\hline AP & $116 \cdot 2$ & $94 \cdot 3,138 \cdot 1$ & 148.9 & $134.4,163.4$ & $142 \cdot 7$ & $127 \cdot 0,158 \cdot 5$ & $155 \cdot 7$ & $135 \cdot 3,176 \cdot 1$ & 0.06 \\
\hline VP & 143.9 & $125 \cdot 4,162 \cdot 4$ & 139.4 & $122 \cdot 8,156 \cdot 0$ & $148 \cdot 3$ & $132 \cdot 4,164 \cdot 1$ & 141.6 & $122 \cdot 9,160 \cdot 3$ & 0.99 \\
\hline \multicolumn{10}{|c|}{ Hispanics } \\
\hline TP & $122 \cdot 1$ & $109 \cdot 7,134.6$ & $123 \cdot 1$ & $114 \cdot 1,132 \cdot 1$ & $129 \cdot 3$ & $120 \cdot 5,138 \cdot 2$ & $124 \cdot 3$ & $113 \cdot 1,135 \cdot 4$ & 0.68 \\
\hline AP & $116 \cdot 3$ & $104 \cdot 4,128 \cdot 3$ & $128 \cdot 8$ & $119 \cdot 4,138 \cdot 1$ & $125 \cdot 7$ & $117 \cdot 0,134 \cdot 4$ & $128 \cdot 8$ & $117 \cdot 3,140 \cdot 3$ & 0.27 \\
\hline VP & 127.7 & $116 \cdot 8,138 \cdot 6$ & $124 \cdot 1$ & $115 \cdot 3,133 \cdot 0$ & $124 \cdot 8$ & $116 \cdot 2,133 \cdot 3$ & 123.6 & $112 \cdot 6,134.6$ & 0.68 \\
\hline
\end{tabular}

Q, quartile; TP, total protein; AP, animal protein; VP, vegetable protein.

"Upper and lower limits of each race/ethnicity-and sex-specific quartile are shown in Table 2.

†From the trend test for the median of bone mineral density in each quartile.

¥Adjusted for age, BMI, physical activity, sedentariness score, smoking, educational level, hormone therapy use (if applicable), age at menopause (if applicable), and intakes of total energy, dietary carbohydrate as a percentage of energy, Ca, P, Mg and alcohol.

$\S$ TP model additionally adjusted for vegetable protein intake as a percentage of energy.

\| TP model additionally adjusted for animal protein intake as a percentage of energy. 
Table 4. Adjusted robust regression coefficient for Z-score of lumbar spine trabecular volumetric bone mineral density among women (highest quartile $v$. lowest quartile of protein intake)

( $\beta$ and $95 \%$ confidence intervals, $n$ 801)

\begin{tabular}{lrrr}
\hline & $\beta$ & $95 \% \mathrm{Cl}$ & $P^{\star}$ \\
\hline Total protein $\dagger$ & & & \\
$\quad$ White & -0.06 & $-0.43,0.31$ & 0.75 \\
Chinese & -0.07 & $-0.80,0.65$ & 0.84 \\
Black & 0.35 & $-0.18,0.88$ & 0.20 \\
$\quad$ Hispanic & 0.16 & $-0.36,0.68$ & 0.55 \\
Animal protein & & & \\
$\quad$ White & -0.13 & $-0.52,0.26$ & 0.51 \\
Chinese & -0.65 & $-1.33,0.03$ & 0.06 \\
Black & 0.29 & $-0.34,0.92$ & 0.37 \\
Hispanic & 0.40 & $-0.16,0.96$ & 0.16 \\
Vegetable protein§ & & & \\
White & 0.44 & $0.07,0.81$ & 0.02 \\
$\quad$ Chinese & 0.22 & $-0.39,0.82$ & 0.49 \\
Black & -0.36 & $-0.88,0.16$ & 0.27 \\
Hispanic & 0.06 & $-0.38,0.51$ & 0.90 \\
\hline
\end{tabular}

${ }^{*}$ From adjusted robust regression analyses.

†Adjusted for age, BMI, physical activity, sedentariness score, smoking, educational level, hormone therapy use, age at menopause, and intakes of total energy, dietary carbohydrate as a percentage of energy, $\mathrm{Ca}, \mathrm{P}, \mathrm{Mg}$ and alcohol.

$\ddagger$ Total protein model additionally adjusted for vegetable protein intake as a percentage of energy.

$\S$ Total protein model additionally adjusted for animal protein intake as a percentage of energy.

among non-Hispanic white women, but their populations were younger and more physically active. A study conducted by Whiting et al. ${ }^{(45)}$ has also shown a significant correlation between total protein intake and BMD in men but did not control for important potential confounders, such as age and physical activity. In a cross-sectional survey, Rapuri et al. ${ }^{(46)}$ reported that women in the highest and second highest quartiles of total protein intake had higher lumbar spine BMD, compared with their counterparts in the lowest quartile; however, this association was not significant in their longitudinal analysis. Darling et al. ${ }^{(4)}$ conducted a meta-analysis of randomised controlled trials and found that participants on protein supplementation had a slightly higher lumbar spine areal BMD compared with those on placebo supplementation, with a weighted mean difference of 0.02 (95\% CI 0.00 , $0.04) \mathrm{g} / \mathrm{cm}^{2}$; however, only three small studies (overall $n$ 110) were included, and sex or racial/ethnic effects were not evaluated. The present results suggest that BMD is not adversely affected by high-protein diets, and build on these prior findings by suggesting that the relationship between dietary protein and bone density may differ by race/ethnicity and protein source, which may partially account for conflicting results.

With regard to the initial findings of vegetable protein intake and bone health in non-Hispanic white women, a cross-sectional study examining the association among 560 white women enrolled in a managed care organisation has shown the same trend: women in a higher tertile of vegetable protein intake had a higher BMD at each skeletal site ( $P$ for trend: 0.03 for hip, 0.10 for lumbar spine and 0.04 for whole body) than did women consuming less vegetable protein ${ }^{(48)}$. Among elderly Chinese women, no association between vegetable protein intake and lumbar spine BMD was identified in one study ${ }^{(49)}$, while another ${ }^{(24)}$ found that vegetable protein intake was positively associated with lumbar BMD in postmenopausal Taiwanese vegetarian women, a population with very different dietary characteristics than the present study population. However, none of the aforementioned results were adjusted for the number of comparisons. Although the basis for these ethnic differences is not clear, the findings as a whole support a neutral or beneficial role of dietary protein on body mineral density.

Mechanisms by which protein intake may influence bone health include increasing $\mathrm{Ca}$ re-absorption or regulating plasma insulin-like growth factor-1 that increases bone formation $^{(5)}$. Isoflavones that are usually contained in plantbased protein diets may influence bone health, although their protective effects as dietary supplements have not been consistently shown in data from randomised controlled trials $^{(50,51)}$. In contrast, protein intake releases protons during the oxidation of sulphur-containing amino acids, and thus lowers $\mathrm{pH}$ and increases bone dissolution ${ }^{(10,11,52)}$. Because animal protein is rich in acidifying amino acids such as cysteine and methionine, whereas vegetable protein is not, the effect of protein intake on BMD could be different by protein source. However, the differences between racial/ ethnic groups could also be due to differences in physiological characteristics and the amount and duration of protein intake.

The results should be interpreted with caution as conclusions from the present study are limited by the following factors. First, this is an observational study so residual confounding cannot be excluded. Second, dietary assessment methods usually include measurement error that might affect both accuracy and precision of study results. Measurement error in the assessment of protein intake is likely to be nondifferential because diet was measured before data collection of lumbar spine vBMD. Third, the type 2 error rate may be increased in some sex- and race/ethnicity-specific subgroups due to reduced sample sizes. However, we conducted power analysis and found at least $80 \%$ power to detect an association in each racial/ethnic group with the exception of $76 \%$ power among Chinese-American women. Fourth, we lacked data on Vitamin D supplementation, medicines for osteoporosis, vBMD on other bone sites, and biochemical markers of bone health. Finally, our findings may not be directly applicable to the elderly because elderly adults require more protein consumption than those in late middle age (mean age of the study population 62 years) in order to maintain $\mathrm{N}$ equilibrium, heal wounds or pressure ulcers, fight infections, or build new tissue to replace normal losses. Recent evidence suggests that the minimum requirement of dietary protein is $1.0 \mathrm{~g} / \mathrm{kg}$ body weight among elders, when compared with $0.8 \mathrm{~g} / \mathrm{kg}$ recommended for middle-aged adults $^{(53)}$. The findings may also not apply to athletes due mainly to increased requirements for protein with high levels of physical activity ${ }^{(54)}$.

The present study also has several strengths that lend confidence to these findings. First, we examined vBMD in a well-characterised sample of multiple ethnicities, which enabled us to investigate and compare the association in 
participants of diverse racial and ethnic origins. Second, MESA followed a stringent protocol, and collected measures using standardised procedures to increase the measurement validity and reliability. Third, validated questionnaires were used for the assessment of habitual dietary intake and confounding variables. Fourth, we adjusted for multiple comparisons to better control type 1 error rates. In addition, information on a variety of potential confounders, including physical activity, BMI, smoking, alcohol consumption, hormone replacement therapy and other dietary factors, was available for adjustment.

In summary, data from the present large, multi-ethnic, population-based study suggest that a higher level of protein intake, when substituted for fat, is not associated with poor bone health among late middle-aged men and women. On the contrary, a higher intake of vegetable protein may be beneficial for bone health among middle-aged non-Hispanic white women. Differences in the relationship between protein source and race/ethnicity of study populations may in part explain inconsistent findings reported previously. Given the prevalence of low bone mass among older women, these results have important public health implications. Additional studies with more detailed dietary assessments are needed to confirm these findings and to examine bone formation or reabsorption markers that may clarify the potential mechanisms for racial/ethnic differences in bone health.

\section{Supplementary material}

To view supplementary material for this article, please visit http://dx.doi.org/10.1017/S0007114514002220

\section{Acknowledgements}

The authors thank the other investigators, the staff and the participants of the MESA study for their valuable contribution. A full list of participating MESA investigators and institutions can be found at http://www.mesa-nhlbi.org

The present study was supported by contracts N01-HC95159 through N01-HC-95166 and N01-HC-95169 from the National Heart, Lung, and Blood Institute (NHLBI) for the collection of data in the MESA study. L. A. B. was supported in part by K08 HL091108 from the NHLBI and in part by P20RR017659 from the National Center for Research Resources (NCRR) through the Tulane Hypertension and Renal Center of Excellence. Both NHLBI and NCRR are components of the National Institutes of Health (NIH). The contents herein are solely the responsibility of the authors and do not necessarily represent the official view of NHLBI, NCRR or NIH.

The authors' responsibilities are as follows: T. H. and L. A. B. contributed to the study concept and design and analysis and interpretation of the data; D. R. J. and M. H. C. contributed to the acquisition of the data; N. J. R., J. A. N., J. A. H., L. M. S., J. H., D. R. J., M. H. C. and L. A. B. provided critical revision of the manuscript for important intellectual content; T. H. contributed to the drafting of the manuscript; L. A. B. had full access to all of the data in the study, and responsibility for the integrity of the data and the accuracy of the data analysis.

The authors declare that they have no conflicts of interest.

\section{References}

1. National Osteoporosis Foundation (2002) America's Bone Health: The State of Osteoporosis and Low Bone Mass in Our Nation. Washington, DC: National Osteoporosis Foundation.

2. Ray NF, Chan JK, Thamer M, et al. (1997) Medical expenditures for the treatment of osteoporotic fractures in the United States in 1995: report from the National Osteoporosis Foundation. J Bone Miner Res 12, 24-35.

3. Riggs BL \& Melton LJ 3rd (1995) The worldwide problem of osteoporosis: insights afforded by epidemiology. Bone 17, Suppl. 5, 505S-511S.

4. Halton TL \& Hu FB (2004) The effects of high protein diets on thermogenesis, satiety and weight loss: a critical review. J Am Coll Nutr 23, 373-385.

5. Ginty F (2003) Dietary protein and bone health. Proc Nutr Soc 62, 867-876.

6. Kerstetter JE \& Allen LH (1990) Dietary protein increases urinary calcium. J Nutr 120, 134-136.

7. Hunt JR, Johnson LK \& Fariba Roughead ZK (2009) Dietary protein and calcium interact to influence calcium retention: a controlled feeding study. Am J Clin Nutr 89, 1357-1365.

8. Abelow BJ, Holford TR \& Insogna KL (1992) Cross-cultural association between dietary animal protein and hip fracture: a hypothesis. Calcif Tissue Int 50, 14-18.

9. Frassetto LA, Todd KM, Morris RC, et al. (2000) Worldwide incidence of hip fracture in elderly women: relation to consumption of animal and vegetable foods. $J$ Gerontol $A$ Biol Sci Med Sci 55, M585-M592.

10. Thorpe M, Mojtahedi MC, Chapman-Novakofski K, et al. (2008) A positive association of lumbar spine bone mineral density with dietary protein is suppressed by a negative association with protein sulfur. J Nutr 138, 80-85.

11. Sellmeyer DE, Stone KL, Sebastian A, et al. (2001) A high ratio of dietary animal to vegetable protein increases the rate of bone loss and the risk of fracture in postmenopausal women. Study of Osteoporotic Fractures Research Group. Am J Clin Nutr 73, 118-122.

12. Feskanich D, Willett WC, Stampfer MJ, et al. (1996) Protein consumption and bone fractures in women. Am J Epidemiol 143, 472-479.

13. Weikert C, Walter D, Hoffmann K, et al. (2005) The relation between dietary protein, calcium and bone health in women: results from the EPIC-Potsdam cohort. Ann Nutr Metab 49, 312-318.

14. Meyer HE, Pedersen JI, Loken EB, et al. (1997) Dietary factors and the incidence of hip fracture in middle-aged Norwegians. A prospective study. Am J Epidemiol 145, 117-123.

15. Munger RG, Cerhan JR \& Chiu BC (1999) Prospective study of dietary protein intake and risk of hip fracture in postmenopausal women. Am J Clin Nutr 69, 147-152.

16. Hannan MT, Tucker KL, Dawson-Hughes B, et al. (2000) Effect of dietary protein on bone loss in elderly men and women: the Framingham Osteoporosis Study. J Bone Miner Res 15, 2504-2512.

17. Promislow JH, Goodman-Gruen D, Slymen DJ, et al. (2002) Protein consumption and bone mineral density in the elderly: the Rancho Bernardo Study. Am J Epidemiol 155, 636-644.

18. Key TJ, Appleby PN, Spencer EA, et al. (2007) Calcium, diet and fracture risk: a prospective study of 1898 incident fractures among 34696 British women and men. Public Health Nutr 10, 1314-1320.

19. Henderson NK, Price RI, Cole JH, et al. (1995) Bone density in young women is associated with body weight and muscle 
strength but not dietary intakes. J Bone Miner Res 10 384-393.

20. Horiuchi T, Onouchi T, Takahashi M, et al. (2000) Effect of soy protein on bone metabolism in postmenopausal Japanese women. Osteoporos Int 11, 721-724.

21. Michaelsson K, Holmberg L, Mallmin H, et al. (1995) Diet, bone mass, and osteocalcin: a cross-sectional study. Calcif Tissue Int 57, 86-93.

22. Wang MC, Luz Villa M, Marcus R, et al. (1997) Associations of vitamin $\mathrm{C}$, calcium and protein with bone mass in postmenopausal Mexican American women. Osteoporos Int 7, 533-538.

23. Kumar A, Mittal S, Orito S, et al. (2010) Impact of dietary intake, education, and physical activity on bone mineral density among North Indian women.J Bone Miner Metab 28, 192-201.

24. Chiu JF, Lan SJ, Yang CY, et al. (1997) Long-term vegetarian diet and bone mineral density in postmenopausal Taiwanese women. Calcif Tissue Int 60, 245-249.

25. Schousboe JT, Shepherd JA, Bilezikian JP, et al. (2013) Executive summary of the 2013 International Society for Clinical Densitometry Position Development Conference on bone densitometry. J Clin Densitom 16, 455-466.

26. Papaioannou A, Morin S, Cheung AM, et al. (2010) 2010 clinical practice guidelines for the diagnosis and management of osteoporosis in Canada: summary. CMAJ 182, 1864-1873.

27. Cauley JA, Blackwell T, Zmuda JM, et al. (2010) Correlates of trabecular and cortical volumetric bone mineral density at the femoral neck and lumbar spine: the osteoporotic fractures in men study (MrOS). J Bone Miner Res 25, 1958-1971.

28. Roux JP, Wegrzyn J, Arlot ME, et al. (2010) Contribution of trabecular and cortical components to biomechanical behavior of human vertebrae: an ex vivo study. $J$ Bone Miner Res 25, 356-361.

29. Bild DE, Bluemke DA, Burke GL, et al. (2002) Multi-ethnic study of atherosclerosis: objectives and design. Am J Epidemiol 156, 871-881.

30. Hyder JA, Allison MA, Wong N, et al. (2009) Association of coronary artery and aortic calcium with lumbar bone density: the MESA Abdominal Aortic Calcium Study. Am J Epidemiol 169, 186-194.

31. Cann CE (1988) Quantitative CT for determination of bone mineral density: a review. Radiology 166, 509-522.

32. Carr JJ, Nelson JC, Wong ND, et al. (2005) Calcified coronary artery plaque measurement with cardiac CT in populationbased studies: standardized protocol of Multi-Ethnic Study of Atherosclerosis (MESA) and Coronary Artery Risk Development in Young Adults (CARDIA) study. Radiology 234, 35-43.

33. Detrano RC, Anderson M, Nelson J, et al. (2005) Coronary calcium measurements: effect of CT scanner type and calcium measure on rescan reproducibility - MESA study. Radiology 236, 477-484.

34. Nettleton JA, Rock CL, Wang Y, et al. (2009) Associations between dietary macronutrient intake and plasma lipids demonstrate criterion performance of the Multi-Ethnic Study of Atherosclerosis (MESA) food-frequency questionnaire. Br J Nutr 102, 1220-1227.

35. Block G, Woods M, Potosky A, et al. (1990) Validation of a self-administered diet history questionnaire using multiple diet records. J Clin Epidemiol 43, 1327-1335.

36. Willett WC (2012) Reproducibility and validity of food frequency questionnaires. In Nutritional Epidemiology, pp. 96-141. New York: Oxford University Press.
37. Lutsey PL, Jacobs DR Jr, Kori S, et al. (2007) Whole grain intake and its cross-sectional association with obesity, insulin resistance, inflammation, diabetes and subclinical CVD: The MESA Study. Br J Nutr 98, 397-405.

38. Levey AS, Stevens LA, Schmid CH, et al. (2009) A new equation to estimate glomerular filtration rate. Ann Intern Med 150, 604-612.

39. Benjamini Y \& Hochberg Y (1995) Controlling the false discovery rate: a practical and powerful approach to multiple testing. J R Stat Soc B 57, 289-300.

40. Dawson-Hughes B \& Harris SS (2002) Calcium intake influences the association of protein intake with rates of bone loss in elderly men and women. Am J Clin Nutr $\mathbf{7 5}$, $773-779$.

41. Roughead ZK, Johnson LK, Lykken GI, et al. (2003) Controlled high meat diets do not affect calcium retention or indices of bone status in healthy postmenopausal women. J Nutr 133, 1020-1026.

42. Cooper C, Atkinson EJ, Hensrud DD, et al. (1996) Dietary protein intake and bone mass in women. Calcif Tissue Int $\mathbf{5 8}, 320-325$.

43. Teegarden D, Lyle RM, McCabe GP, et al. (1998) Dietary calcium, protein, and phosphorus are related to bone mineral density and content in young women. Am J Clin Nutr 68, 749-754.

44. Quintas ME, Ortega RM, Lopez-Sobaler AM, et al. (2003) Influence of dietetic and anthropometric factors and of the type of sport practised on bone density in different groups of women. Eur J Clin Nutr 57, Suppl. 1, S58-S62.

45. Whiting SJ, Boyle JL, Thompson A, et al. (2002) Dietary protein, phosphorus and potassium are beneficial to bone mineral density in adult men consuming adequate dietary calcium. J Am Coll Nutr 21, 402-409.

46. Rapuri PB, Gallagher JC \& Haynatzka V (2003) Protein intake: effects on bone mineral density and the rate of bone loss in elderly women. Am J Clin Nutr 77, 1517-1525.

47. Darling AL, Millward DJ, Torgerson DJ, et al. (2009) Dietary protein and bone health: a systematic review and metaanalysis. Am J Clin Nutr 90, 1674-1692.

48. Beasley JM, Ichikawa LE, Ange BA, et al. (2010) Is protein intake associated with bone mineral density in young women? Am J Clin Nutr 91, 1311-1316.

49. Lau EM, Kwok T, Woo J, et al. (1998) Bone mineral density in Chinese elderly female vegetarians, vegans, lacto-vegetarians and omnivores. Eur J Clin Nutr 52, 60-64.

50. Ricci E, Cipriani S, Chiaffarino F, et al. (2010) Soy isoflavones and bone mineral density in perimenopausal and postmenopausal Western women: a systematic review and meta-analysis of randomized controlled trials. $J$ Womens Health (Larchmt) 19, 1609-1617.

51. Liu J, Ho SC, Su YX, et al. (2009) Effect of long-term intervention of soy isoflavones on bone mineral density in women: a meta-analysis of randomized controlled trials. Bone 44, 948-953

52. Rizzoli R \& Bonjour JP (2004) Dietary protein and bone health. J Bone Miner Res 19, 527-531.

53. Chernoff R (2004) Protein and older adults. J Am Coll Nutr 23, Suppl. 6, 627S-630S.

54. Phillips SM \& Van Loon LJ (2011) Dietary protein for athletes: from requirements to optimum adaptation. J Sports Sci 29, Suppl. 1, S29-S38. 